\title{
CYTISUS STRIATUS (FABACEAE), NUEVA “RETAMA” ADVENTICIA EN ARGENTINA
}

\author{
Javier G. Puntieri \& Jorge O. Chiapella²
}

\begin{abstract}
${ }^{1}$ Instituto de Investigaciones en Recursos Naturales, Agroecología y Desarrollo Rural (IRNAD-CONICET), Universidad Nacional de Río Negro, Sede Andina, Güemes 383, El Bolsón, CP 8300, Río Negro, Argentina; jpuntieri@unrn.edu.ar (autor corresponsal).

${ }^{2}$ Instituto Multidisciplinario de Biología Vegetal (IMBIV-CONICET), Universidad Nacional de Córdoba, POBox 495, CP 5000, Córdoba, Argentina.
\end{abstract}

\begin{abstract}
Puntieri, J. G. \& J. O. Chiapella. 2019. Cytisus striatus (Fabaceae), a new adventitious "broom" in Argentina. Darwiniana, nueva serie 7(2): 335-341.

The "broom" Cytisus striatus is cited for the first time for Argentina. This species coexists with the similar but more widespread "scotch broom", C. scoparius, frequent in disturbed habitats of Patagonia and temperate regions worldwide. So far, eight ruderal seed-producing individuals of C. striatus have been spotted in Patagonia. The morphological resemblance between $C$. striatus and $C$. scoparius, and the distance between both populations of $C$. striatus found in Argentina up to now (31 km) suggest that this species could be abundant in Andean regions of Patagonia. This paper provides a key for the identification of the brooms of Tribe Cytiseae present in Argentina.
\end{abstract}

Keywords. Broom; exotic shrubs; Fabaceae; Patagonia.

Resumen. Puntieri, J. G. \& J. O. Chiapella. 2019. Cytisus striatus (Fabaceae), nueva "retama" adventicia en Argentina. Darwiniana, nueva serie 7(2): 335-341.

Se cita por primera vez la presencia de la "retama" Cytisus striatus en Argentina. Esta especie coexiste con la muy semejante pero más difundida "retama de escobas", C. scoparius, frecuente en ambientes disturbados de la Patagonia y de otras regiones templadas del mundo. Por el momento se han identificado en Patagonia ocho ejemplares ruderales de C. striatus, todos ellos productores de semillas. La similitud morfológica entre $C$. striatus y $C$. scoparius y la distancia entre las dos poblaciones de C. striatus registradas hasta el momento en Argentina $(31 \mathrm{~km})$ sugieren que esta especie podría ser abundante en la Patagonia andina. Esta publicación aporta una clave para diferenciar las retamas de la tribu Cytiseae presentes en Argentina.

Palabras clave. Arbustos exóticos; Fabaceae; Patagonia; retama.

\section{INTRODUCCIÓN}

El género Cytisus Desf. (Fabaceae, Faboideae, Cytiseae; Lewis et al., 2005) posee su área de distribución natural en la cuenca del mar Mediterráneo. Se trata de plantas leñosas denominadas comúnmente "retamas", con tallos verdes, hojas pequeñas o ausentes, y amplia tolerancia a variaciones ambientales (Bisby, 1981). En Argentina se cultivan como ornamentales varias especies de "retama" del género Cytisus y de otros géneros de la tribu Cytiseae; para algunas de ellas se registran poblaciones espontáneas: Genista tinctoria L. (Neuquén y Río Negro), Genista monspessulana (L.) L.A.S. Johnson, "genista de Montpellier" (Buenos Aires, Chubut y La Pampa), 
Spartium junceum L., "retama española" (Buenos Aires, Entre Ríos, La Pampa y Río Negro) y Cytisus scoparius (L.) Link, "retama" (Buenos Aires, Chubut, Neuquén y Río Negro) (Fortunato, 1984, 2008; Hurrell \& Lahitte, 2002). Esta última ha alcanzado el estatus de invasora en ambientes disturbados del norte de la Patagonia, así como en Australia, Brasil, Canadá, Chile, India, Irán, Nueva Zelanda, Australia, Sudáfrica y los Estados Unidos de América (Matthei, 1995; Fowler et al., 1996; Peterson \& Prasad, 1998; Fogarty \& Facelli, 1999; Talavera, 1999; Bellingham \& Coomes, 2003; Bellingham et al., 2005; Cordero et al., 2016; Talukdar \& Talukdar, 2016). Cytisus scoparius es objeto de programas de control tanto químico como biológico (Williams, 1981; Peterson \& Prasad, 1998; Ketchum \& Rose, 2003). Desde hace varias décadas se ha hecho referencia a la adaptabilidad de esta especie a las condiciones climáticas de la Patagonia andina (Dimitri, 1979; Brion et al., 1988), donde cubre grandes superficies y produce impresionantes floraciones amarillas. Aunque no se dispone de información cuantitativa, las extensas y densas poblaciones ruderales de $C$. scoparius estarían influyendo negativamente sobre la diversidad de las comunidades vegetales nativas, como ocurre en otros países (Bossard \& Rejmánek, 1992; Peterson \& Prasad, 1998; Carter et al., 2019).

En el centro de Chile, en la región de Bío Bío, habita Cytisus striatus (Hill) Rothm. (Matthei, 1995; Rodríguez et al., 2018), una "retama" exótica muy semejante a $C$. scoparius, pero que hasta el momento no había sido registrada para la Argentina. En esta comunicación se informa por primera vez la presencia de poblaciones espontáneas de $C$. striatus en las provincias argentinas de Río Negro (depto. Bariloche) y Chubut (depto. Cushamen) y se suministra una clave para diferenciar las cinco especies de "retamas" de la tribu Cytiseae detectadas hasta el momento en la Patagonia.

\section{MATERIALES Y MÉTODOS}

El material estudiado de C. striatus se recolectó en marzo de 2017 en dos poblaciones distantes $31 \mathrm{~km}$ entre sí, una ubicada en cercanías de la localidad de El Bolsón, departamento
Bariloche, provincia de Río Negro, y la restante en cercanías de la localidad de Epuyén, departamento Cushamen, provincia de Chubut (Figura 1). Se conservan muestras de estas dos colecciones en el herbario BCRU (Thiers, 2019). La morfología de este material fue analizada bajo microscopio estereoscópico (Leica EZ4D, hasta 40x), arribándose a la identificación luego de compararse este material con las descripciones de las especies de Cytiseae de Flora Ibérica (Talavera, 1999) y con las descripciones e imágenes de las especies de Cytisus de la revisión de Auvray y Malécot (2013). Las muestras de C. striatus fueron contrastadas con muestras de $S$. junceum, C. scoparius, G. tinctoria y G. monspessulana pertenecientes al herbario BCRU y de ejemplares asilvestrados presentes en las provincias de Río Negro (las dos primeras especies) y Chubut (las tres últimas). Los ejemplares de $C$. striatus recolectados en El Bolsón y Epuyén fueron comparados con la descripción original de esta especie y con la imagen del lectotipo.

\section{RESULTADOS}

Cytisus striatus (Hill) Rothm., Feddes Repert. Spec. Nov. Regni Veg. 53: 149. 1944. Genista striata Hill., Veg. Syst. 13 (Appendix): 63, pl. 13. 1768. Spartium striatum (Hill) Samp., Herb. Port.: 145. 1913. Sarothamnus striatus (Hill) Samp., Bol. Soc. Brot., sér. 2, 10: 226. 1935. TIPO: Lámina13, en J. Hill, Veg. Syst. 13: 63. 1768, lectotipo designado por Auvray \& Malécot (2013: 101). Figuras 2 y 3.

Arbusto o arbolito hasta $3 \mathrm{~m}$ de altura; ramas seríceas o glabras, con 7-10 crestas longitudinales redondeadas, separadas por surcos puberulentos. Hojas compuestas, 1-folioladas y 3-folioladas, con estípulas muy pequeñas $(<1 \mathrm{~mm})$, suborbiculares, poco evidentes o ausentes. Hojas 1-folioladas sésiles o con pecíolo muy corto y lámina obovada, de 3-10 × 2,5-4 mm. Hojas 3-folioladas con pecíolo largo, aplanado, y folíolos oblanceolados o elípticos, de $6-12 \times 1,5-5 \mathrm{~mm}$, el central algo mayor que los laterales. Superficie de las hojas con pelos adpresos más abundantes en el envés de los folíolos. Flores 1-2, axilares; pedicelos algo pilosos. 


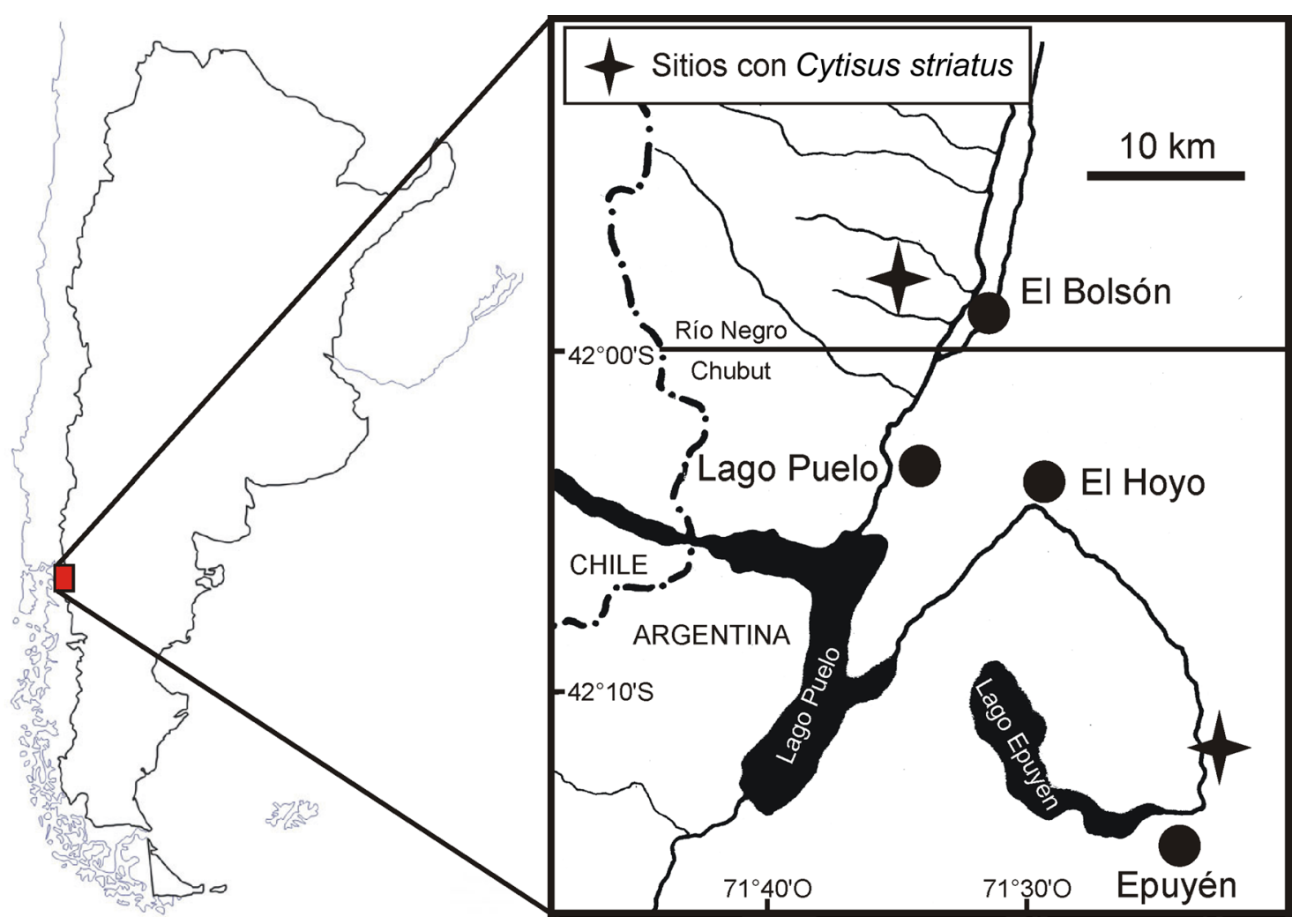

Fig. 1. Sitios de colección de Cytisus striatus en las provincias de Río Negro y Chubut. Las estrellas señalan las localizaciones de las poblaciones reportadas. Se indican con círculos negros las localidades más cercanas a dichas poblaciones.

Cáliz de $7 \mathrm{~mm}$, bilabiado, con el labio superior bidentado y el inferior tridentado, este último algo más largo; superficie con pelos seríceos. Corola amarilla, papilionada; estandarte brevemente unguiculado, con lámina suborbicular, ca.17 $\times 19 \mathrm{~mm}$; alas oblongas, de $19 \times 8 \mathrm{~mm}$, unguiculadas y semisagitadas; quilla formada por 2 pétalos obovados y curvados, ca. $20 \mathrm{~mm}$, unguiculados y semisagitados, con un breve espolón en la base de la lámina. Androceo monadelfo, con 2 estambres largos, 2 medianos y 6 cortos; columna de filamentos de $9 \times 3,5 \mathrm{~mm}$. Gineceo de $25 \mathrm{~mm}$; ovario densamente pubescente; estilo aplanado, distalmente engrosado y curvado en su extremo. Fruto legumbre, albo-pubescente, curvada, de hasta $35 \times 13 \mathrm{~mm}$, dehiscente, conteniendo hasta 8 semillas pardusco-rojizas de 3-4 mm, con carúncula blanco-amarillenta. Número cromosómico 2n=46, 48 (Talavera, 1999).
Nombres vulgares. "Escoba", “escobón”, "retama", "retama de escobas" (Talavera, 1999).

Distribución y hábitat. Se la considera una especie nativa del oeste de la Península Ibérica (España y Portugal) y del noroeste de África (Marruecos), e introducida en el resto de Europa (Francia, Reino Unido), en América del Norte (Talavera, 1999) y en Chile (Matthei, 1995). Habita en sitios abiertos, disturbados y en claros de bosques (Talavera, 1999). En la Argentina se ha registrado su presencia en dos sitios con vegetación ruderal. Uno de ellos se encuentra en el paraje Mallín Ahogado, perteneciente al ejido de El Bolsón (depto. Bariloche, provincia de Río Negro), al borde del camino que une el área recreativa Cascada Escondida (bajo jurisdicción del INTA Bariloche) con la ruta 40 (41 $55^{\prime}$ ' 19 " S, $71^{\circ} 32^{\prime} 20^{\prime \prime} \mathrm{O}, 458 \mathrm{~m}$ s.m.). 


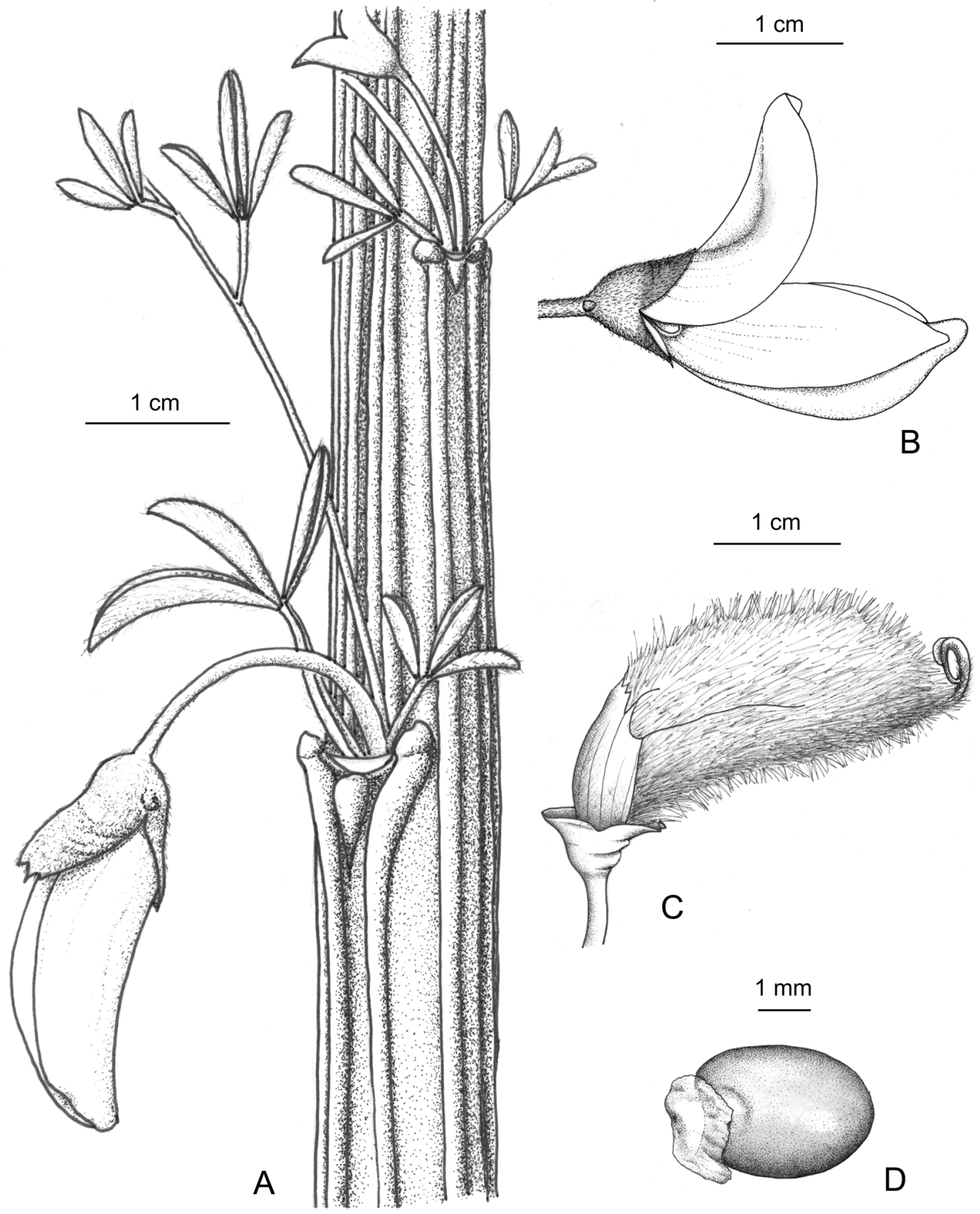

Fig. 2. Cytisus striatus. A, porción distal de un tallo florecido. B, detalle de flor abierta. C, fruto. D, semilla. Ilustración: J. Puntieri (de Puntieri 921, BCRU). 
El segundo sitio abarca los bordes del camino de acceso a la localidad de Epuyén (depto. Cushamen, provincia de Chubut) y de la ruta 40 (42 12 ' 03 " S, $71^{\circ} 23^{\prime} 44^{\prime \prime}$ O, 375 m s.n.m.). En el primero de estos sitios se encontraron tres y en el segundo cinco ejemplares adultos, todos ellos de entre 1,5 y $2,5 \mathrm{~m}$ de altura y de entre 7 y $12 \mathrm{~cm}$ de diámetro del tallo al nivel del suelo; la distancia entre individuos fue siempre superior a 10 metros. Al momento de realizarse el primer registro, todos estos ejemplares se encontraban en proceso de liberación de las semillas producidas al final de la estación de crecimiento 2016-17 y tenían varios de sus frutos en pie. Se comprobó la viabilidad de estas semillas mediante una prueba de germinación luego de retirada la carúncula (J. Puntieri datos no publicados). En noviembre de 2017 se observaron esos ejemplares en plena floración (Figura 3A). Las comunidades ruderales donde se encontraron los ejemplares de C. striatus son el resultado de la alteración de bosques nativos de Austrocedrus chilensis (D. Don) Pic. Serm. \& Bizarri. Actualmente están dominadas por C. scoparius, Rosa rubiginosa L. y Juniperus communis L. en el estrato arbustivo y por $A$. chilensis, Pseudotsuga menziesii (Mirb.) Franco y Pinus contorta Douglas ex Loudon en el arbóreo. En base a las indagaciones realizadas por los autores en los principales viveros registrados entre el noroeste de Chubut y el sudoeste de Río Negro, C. striatus no es ni ha sido cultivada en esa región.
Observaciones. Cytisus striatus y $C$. scoparius se asemejan en su morfología vegetativa $\mathrm{y}$ tamaño, morfología floral y fenología. Las principales características que permiten diferenciar estas dos especies a campo son: (1) el número y la forma de las crestas y surcos de los tallos ( 5 crestas aplanadas en C. scoparius, 7-10 crestas redondeadas en C. striatus)(Fig. 3B), (2) el aroma de las flores (agradable en $C$. scoparius, desagradable en $C$. striatus), (3) el tamaño y color de los frutos maduros (negros y de más de $5 \mathrm{~cm}$ de largo en C. scoparius; albo-pubescentes y de menos de $4 \mathrm{~cm}$ en $C$. striatus) y (4) el número de semillas por fruto (20 ó más en C. scoparius, hasta 8 en $C$. striatus). El color y la longitud de los frutos maduros son los rasgos distintivos más evidentes cuando se observa las plantas a varios metros de distancia. El número de ejemplares de C. striatus encontrados hasta el momento en Chile y Argentina es notablemente inferior al número de ejemplares de C. scoparius (o de otras "retamas" de la tribu Cytiseae) presentes en esos países (Rodríguez et al., 2018). Se necesitan estudios más específicos y detallados para determinar si la distribución de C. striatus en Argentina es mayor de la que se informa aquí.

\section{Material examinado}

ARGENTINA. Río Negro. Depto. Bariloche: Mallín Ahogado, camino a Cascada Escondida, 5III-2017, Puntieri 920 (BCRU). Chubut. Depto. Cushamen: Epuyén, acceso norte a Epuyén desde ruta nacional 40, 1-XII-2017, Puntieri 921 (BCRU).

\section{Clave de diferenciación de las "retamas" de la tribu Cytiseae asilvestradas en el norte de Patagonia}

1. Inflorescencias en general 1-2-floras

1. Inflorescencias plurifloras

2(1). Tallos jóvenes con 5 crestas longitudinales y surcos glabros; frutos de más de $5 \mathrm{~cm}$ de longitud, negros y con pubescencia laxa a la madurez Cytisus scoparius

2. Tallos jóvenes con 7-10 crestas longitudinales y surcos puberulentos; frutos de menos de $5 \mathrm{~cm}$ de longitud, con pubescencia densa de pelos blancos a la madurez Cytisus striatus 3(1). Inflorescencias de más de $30 \mathrm{~cm}$ de longitud, que superan el follaje en altura; ramas estriadas o lisas .....

Spartium junceum

3. Inflorescencias de menos de $20 \mathrm{~cm}$ de longitud, poco elevadas sobre el follaje; ramas con crestas longitudinales ......... 4 4(3). Inflorescencias corimbiformes; plantas adultas de más de 1,5 m de altura ...................... Genista monspessulana 4. Inflorescencias racemiformes; plantas adultas hasta 1,5 $\mathrm{m}$ de altura Genista tinctoria 

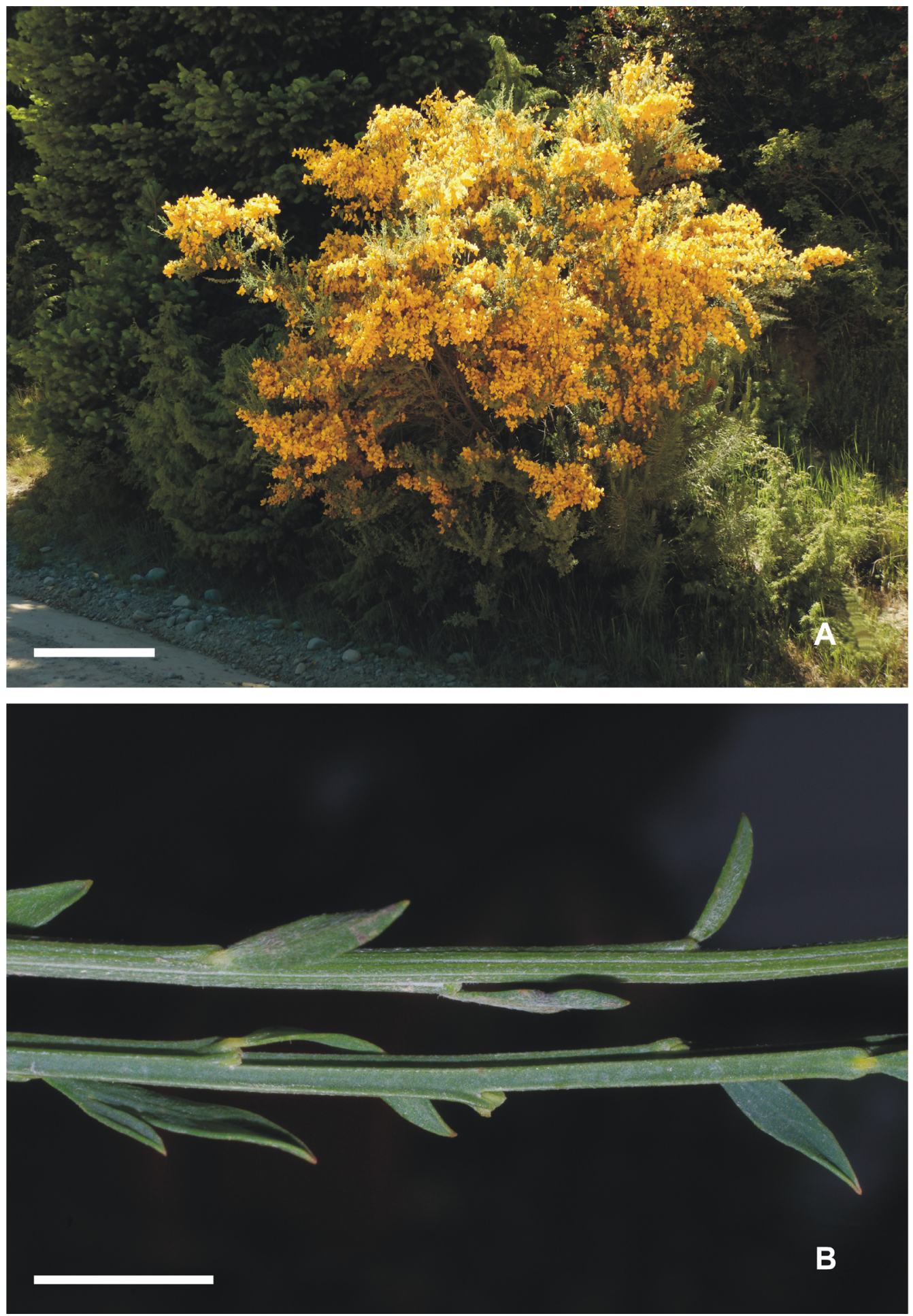

Fig. 3. A, un ejemplar de C. striatus en plena floración (Mallín Ahogado, El Bolsón). B, porciones de tallo de C. striatus (arriba) y C. scoparius (abajo). Escalas $=\mathrm{A}: 50 \mathrm{~cm}$; B: $1 \mathrm{~cm}$. Figura en color en la versión en línea http://www.ojs.darwin.edu.ar/index.php/darwiniana/article/view/837/1166 


\section{J. G. PUNTIERI \& J. O. CHIAPELLA. Cytisus striatus, nueva retama en Argentina}

\section{AGRADECIMIENTOS}

Los autores agradecen a Laura D'Atri por su colaboración en la búsqueda de ejemplares de C. striata, y a los revisores de la primera versión del manuscrito que contribuyeron en mejorarla.

\section{BIBLIOGRAFÍA}

Auvray, G. \& V. Malécot. 2013. A revision of Cytisus sections alburnoides, spartopsis and verzinum (Genisteae, Fabaceae). Edinburgh Journal of Botany 70(1): 61-120.

Bellingham, P. J. \& D. A. Coomes. 2003. Grazing and community structure as determinants of invasion success by Scotch broom in a New Zealand montane shrubland. Diversity and Distributions 9: 19-28.

Bellingham, P. J., D. A. Peltzer \& L. R. Walker. 2005. Contrasting impacts of a native and an invasive exotic shrub on floodplain succession. Journal of Vegetation Science 16: 135-142.

Bisby, F. A. 1981. Genisteae (Adans.) Benth., en R. M. Polhill \& P. H.Raven (eds.), Advances in Legume Systematics 1, pp. 409-425. Kew: Royal Botanic Gardens.

Bossard, C. C. \& M. Rejmánek. 1992. Why have green stems. Functional Ecology 6:197-205.

Brion, C., J. G. Puntieri, D. Grigera \& S. Calvelo. 1988. Flora de Puerto Blest y sus alrededores. Bariloche: EDUCO, Universidad Nacional del Comahue.

Carter, D. R., R. A. Slesak, T. B. Harrington, D. H. Peter \& A. W. D'Amato. 2019. Scotch broom (Cytisus scoparius) modifies microenvironment to promote nonnative plant communities. Biological Invasions 21: 1055-1073

Cordero, R. L., F. P. Torchelsen, G. E. Overbeck \& M. Anand. 2016. Cytisus scoparius (Fam. Fabaceae) in southern Brazil - first step of an invasion process? Anais da Academia Brasileira de Ciências 88: 149-154.

Dimitri, M. J. 1979. Sarothamnus, en L. R. Parodi (ed.), Enciclopedia Argentina de Agricultura y Jardinería, $3^{\text {ra }}$ ed., actualizada por M. J. Dimitri, vol. 1, pp. 517. Buenos Aires: ACME.

Fogarty, G. \& J. M. Facelli. 1999. Growth and competition of Cytisus scoparius, an invasive shrub, and Australian native shrubs. Plant Ecology 144: 27-35.

Fortunato, R. H. 1984. Sarothamnus Wimm., en M. N. Correa (ed.), Flora Patagónica. Colección Cientifica del Instituto Nacional de Tecnología Agropecuaria 8(IVb): 267-268.
Fortunato, R. H. 2008. Fabaceae, en F. O. Zuloaga, F., O. Morrone. \& M. J. Belgrano (eds.). Catálogo de las Plantas Vasculares del Cono Sur (Argentina, Sur de Brasil, Chile, Paraguay y Uruguay). Monographs in Systematic Botany from the Missouri Botanical Garden 107(2): 2078-2286.

Fowler, S. V., H. M. Harman, J. Memmott, Q. Paynter, R. Shaw, A. W. Sheppard \& P. Syrett. 1996. Comparing the population dynamics of broom, Cytisus scoparius, as a native plant in the United Kingdom and France and as an invasive alien weed in Australia and New Zealand, en V. C. Moran \& J. H. Hoffmann (eds.), Proceedings of the IX International Symposium on Biological Control of Weeds, pp. 19-26. Stellenbosch: University of Cape Town.

Hurrell, J. A. \& H. B. Lahitte. 2002. Biota Rioplatense VII, Leguminosas nativas y exóticas. Buenos Aires: Editorial L.O.L.A. (Literature of Latin America).

Ketchum, J. S. \& R. Rose. 2003. Preventing establishment of exotic shrubs (Cytisus scoparius (L.) Link. and Cytisus striatus (Hill)) with soil active herbicides (hexazinone, sulfometuron, and metsulfuron). New Forests 25: 83-92.

Lewis, G., B. Schrire, B. Mackinder \& M. Lock. 2005. Legumes of the World. Kew: Royal Botanic Gardens.

Matthei, O. 1995. Catálogo de las Malezas que crecen en Chile. Santiago de Chile: Alfabeta Impresores.

Peterson, D. J. \& R. Prasad. 1998. The biology of Canadian weeds. 109. Cytisus scoparius (L.) Link. Canadian Journal of Plant Sciences 78: 497-504.

Rodríguez, R. et al. 2018. Catálogo de las plantas vasculares de Chile. Gayana, Botanica 75(1): 1-430.

Talavera, S. 1999. Cytisus, en S. Castroviejo et al. (eds.), Flora Ibérica, plantas vasculares de la Península Ibérica e Islas Baleares. Vol. VII (I) Leguminosas (partium): 147-182. Madrid: Real Jardín Botánico, CSIC.

Talukdar, D. \& T. Talukdar. 2016. Inventory of invasive alien plants in Bethuadahari wildlife sanctuary in Nadia district, West Bengal, India. Tropical Plant Research 3: 120-130.

Thiers, B. [permanentemente actualizado, consulta 2019]. Index Herbariorum: A global directory of public herbaria and associated staff. New York Botanical Garden's Virtual Herbarium. http://sweetgum.nybg.org/ science/ih/

Williams, P.A. 1981. Aspects of the ecology of broom (Cytisus scoparius) in Canterbury, New Zealand. New Zealand Journal of Botany 19: 31-43. 